\title{
Study of Automobile Suspension System Vibration Characteristics Based on the Adaptive Control Method
}

\author{
Ruqiang Mou and Li Hou \\ School of Manufacturing Science and Engineering, Sichuan University (610065), Chengdu, China \\ Yiqiang Jiang and Yong Zhao \\ School of Leshan Vocational and Technical College(614000), Leshan, China \\ Yongqiao Wei \\ School of Manufacturing Science and Engineering, Sichuan University (610065), Chengdu, China
}

\begin{abstract}
(Received 9 December 2013; accepted 30 May 2014)
An automotive suspension determines both the driving stability and comfort of the vehicle occupants. This paper establishes two kinds of two degrees of freedom for the automobile suspension vibration model and uses the PID controller to establish an automobile suspension adaptive open-loop and closed-loop control system. Respectively, by step interference, white noise and sinusoidal interference for the input, studying the vibration characteristics of the vibration model in the vertical direction. By numerical simulation, we obtain the suspension of the vertical displacement and acceleration-time graphs. The simulation results show that the vibration characteristics of the first model are more in accordance with the actual situation of the car, and the closed-loop control is better than the open-loop control. The adaptive closed-loop control system can reduce the output displacement of automobile suspension to around $1 \%$ of the interference road input displacement. The output acceleration value is small, and the acceleration changes smoothly. The results verify the rationality and validity of the automobile suspension model and adaptive control system, which provides a theoretical foundation for the design and optimization of the automobile suspension system.
\end{abstract}

\section{INTRODUCTION}

Suspensions are an important part of the car chassis as they directly determine the car's ride comfort and handling stability, but because of their high costs and high energy demand, their application is restricted. Hence, the semi-active suspension system is now used in the automobile suspension structure.

Over the years, many studies on suspension systems have been done. ${ }^{1-15}$ Tomoaki Mori ${ }^{1}$ put forward an adaptive damper controller for compensating the nonlinear hysteresis dynamics of the MR damper. Kayhan Gulez, ${ }^{2}$ Ali Ahmed Adam, ${ }^{12}$ and Hua $\mathrm{Li}^{14}$ use an algorithm method to research the nonlinear characteristics for the semi-active suspension of automobiles. Enrico Pellegrini ${ }^{3}$ and Zhao Cheng ${ }^{4}$ build a semi-active suspension system model based on control strategy in order to describe the damper behaviour. Li Shaohua ${ }^{5}$ and Liang Shan ${ }^{6}$ studied the two degrees of freedom 1/4 automobile suspension model; the former used a multi-scale method to study the combination of multi-frequency excitation resonance characteristics, and the latter researched the suspension model chaotic vibration occurring in road roughness excitation. Ren Chenglong ${ }^{7}$ established a single degree of freedom model of automobile suspension under random excitation; the numerical simulation verifies the automobile suspension chaotic vibration characteristic. ${ }^{7,11}$ Jiammin Sun and Qingmei Yang ${ }^{8}$ established a suspension model and analysed the comfort and safety based on the adaptive filter theory. Abu-Khudhair, ${ }^{9}$ Changizi, ${ }^{10}$ and Barr ${ }^{13}$ use a fuzzy logic technique to build the semi-active automobile suspension systems control, which has a significantly fewer number of rules in comparison to existing fuzzy controllers. Hung Yichen ${ }^{15}$ proposes a functionalapproximation based adaptive sliding controller with fuzzy compensation for an active suspension system.

The above studies on automobile suspension do not simultaneously propose two vibration models based on the adaptive control method so they can't prove which model is more suitable for an automobile suspension system. Hence, this paper gives two automobile suspension system vibration models using the adaptive control method and two control algorithms consisting of PID controllers. This paper also studies suspension system vibration characteristics with step input, whitenoise input, and sinusoidal input as the external excitation. By numerical simulation, this study obtains the displacement and acceleration characteristics. Comparing the results of the two models, we find that when examining the engine and the suspension as a whole, the suspension system vibration characteristics more closely match the actual conditions, which is conducive for designing the most appropriate automobile suspension model. 